$11-1-2008$

\title{
On Some Properties of Quasi-Negative-Binomial Distribution and Its Applications
}

Anwar Hassan

University of Kashmir, anwar.hassan2007@gmail.com

Sheikh Bilal

DegreeCollege, sbilal_sbilal@yahoo.com

Follow this and additional works at: http://digitalcommons.wayne.edu/jmasm

Part of the Applied Statistics Commons, Social and Behavioral Sciences Commons, and the Statistical Theory Commons

\section{Recommended Citation}

Hassan, Anwar and Bilal, Sheikh (2008) "On Some Properties of Quasi-Negative-Binomial Distribution and Its Applications," Journal of Modern Applied Statistical Methods: Vol. 7 : Iss. 2 , Article 26.

DOI: $10.22237 /$ jmasm/1225513500

Available at: http://digitalcommons.wayne.edu/jmasm/vol7/iss2/26 


\title{
EARLY SCHOLARS \\ On Some Properties of Quasi-Negative-Binomial Distribution and Its Applications
}

\author{
Anwar Hassan \\ University of Kashmir \\ India
}

\author{
Sheikh Bilal \\ Degree College \\ India
}

The quasi-negative-binomial distribution was applied to queuing theory for determining the distribution of total number of customers served before the queue vanishes under certain assumptions. Some structural properties (probability generating function, convolution, mode and recurrence relation) for the moments of quasi-negative-binomial distribution are discussed. The distribution's characterization and its relation with other distributions were investigated. A computer program was developed using $\mathrm{R}$ to obtain ML estimates and the distribution was fitted to some observed sets of data to test its goodness of fit.

Key words: Simultaneous quasi-negative-binomial distribution, Borel-Tanner distribution, probability generating function, convolution property, characterization, chi-square fitting.

\section{Introduction}

The classical negative binomial distribution has become increasingly popular as a more flexible alternative to the Poisson distribution, especially in cases when it is doubtful whether the strict independence requirements for a Poisson distribution will be satisfied. In a classical negative binomial distribution the probability of success from trial to trial is assumed to be constant, but this assumption holds true only in the case of chance mechanism and is not realistic for many practical situations. Most living beings use past experiences (successes or failures) and wisdom to help determine future strategies to achieve goals, thus, the probability of success or failure does not remain constant. It is generally felt that the probability of a success

Anwar Hassan is a Post Graduate in the Department of Statistics. Research interests: Probability and Lagrangian Probability distributions and Statistical Inference. E-mail: anwar.hassan2007@gmail.com. Sheikh Bilal Ahmad is in the Department of Statistics. Research interests: Probability distributions and Statistical Inference. E-mail: sbilal_sbilal@yahoo.com. depends on the number of previous failures, and the quasi- negative-binomial distribution, as well as other distributions, takes this fact into consideration.

Much work has been done on the quasibinomial distribution, but little has been done on quasi-negative-binomial distribution. The quasinegative-binomial distribution has been obtained in different forms by Janardan (1975), Nandi \& Das (1994), and Sen \& Jain (1996), but has not to date been studied in detail. This article examines various aspects of this distribution. The distribution of the number of customers served in the queuing theory under certain assumptions, which gives rise to a quasinegative-binomial distribution, was derived. It is also shown that the quasi-negative-binomial distribution belongs to a family of Abel series distributions. Some structural properties of the distribution are discussed, along with its relation with some other important distributions, and a characterization of the distribution is provided. A computer program written in $\mathrm{R}$ was developed to obtain ML estimates and the distribution was fitted to a number of data sets to show its superiority over other distributions. 


\section{Methodology}

Quasi-negative-binomial distribution (QNBD) In the theory of queuing, suppose there exists a single queue beginning with r customers. Haight \& Brever (1960) showed that, if it is first assumed that the random arrival time of a customer is at a constant rate $(\lambda)$, and a constant amount of time is devoted to serving each customer $(\beta)$, then the probability distribution of the total number of customers served before the queue vanishes is:

$$
\begin{gathered}
P(X: x)=\frac{r}{(x-r) !} x^{x-r-1}(\lambda \beta)^{x-r} e^{-\lambda \beta x} \\
x=r, r+1, \ldots \ldots .
\end{gathered}
$$

This is known as the Borel-Tanner distribution and it gives the probability of a customer arriving during the period $(t, t+\Delta t)$ as $\lambda(\Delta t)+0(\Delta t)$, by assuming $\lambda$ is constant, where $0(\Delta t)$ is the probability of two or more customers arriving in this period. This assumption, however, is not realistic. The random arrival time of customers is not at a constant rate, it varies from interval to interval of equal length. In order to make the formula more flexible it is allowed to vary in different intervals of equal length with a constant amount of time $(\beta)$ spent serving each customer. Thus gives the probability distribution of total number of customers served before the queue vanishes as:

$$
\begin{aligned}
& P(X: x)= \\
& E\left[\frac{r}{(x-r) !} x^{x-r-1}(\lambda \beta)^{x-r} e^{-\lambda \beta x}\right]
\end{aligned}
$$

where expectation is to be taken over $\lambda$. Suppose that the distribution of $\lambda$ is a gamma variate with parameters $(a, b)$, then the above equation becomes:

$$
\begin{aligned}
& P(X=x)=\frac{r}{(x-r) !} x^{x-r-1} \\
& \beta^{x-r} \frac{b^{a}}{\Gamma(a)} \int_{0}^{\infty} \lambda^{a+x-r-1} e^{-\lambda(b+\beta x)} d \lambda
\end{aligned}
$$

$$
=\frac{\beta r}{(x-r) !}(\beta x)^{x-r-1} \frac{b^{a}}{\Gamma(a)} \frac{\Gamma(a+x-r)}{(b+\beta x)^{a+x-r}}
$$

Taking $x=x+r$, that is, starting with an idle queue the probability distribution becomes:

$$
\begin{gathered}
P(X: x)=\frac{\Gamma(a+x)}{\Gamma(a) x !} \frac{(\beta r) b^{a}(\beta r+\beta x)^{x-1}}{(b+\beta r+\beta x)^{a+x}} \\
x=0,1,2, \ldots \ldots \ldots \ldots \\
\quad=\left(\begin{array}{c}
a+x-1 \\
x
\end{array}\right) \frac{\theta_{1}\left(\theta_{1}+\theta_{2} x\right)^{x-1}}{\left(1+\theta_{1}+\theta_{2} x\right)^{a+x}} \\
x=0,1,2, \ldots . .
\end{gathered}
$$

where $\beta r b^{-1}=\theta_{1}, \beta b^{-1}=\theta_{2}$. The distribution represented by (2.4) is a quasi-negative-binomial distribution (QNBD). Hence, the distribution of the total number of customers served before the queue vanishes, assuming a start with an idle queue wherein the random arrival time of customers follows a gamma distribution and the time occupied in serving each customer is constant, is a QNBD.

Equation 2.3 clearly suggests that the quasi-negative-binomial distribution is a mixture of the Borel-Tanner distribution (2.1) with gamma $\gamma(a, b)$ as the mixing distribution. Another way of obtaining the QNBD (2.4) is to compound the restricted generalized Poisson model $(\theta, \alpha \theta)$ with the gamma distribution $\gamma(a, b)$, where $\theta_{1}=b^{-1}$ and $\theta_{2}=\alpha b^{-1}$. This is the method employed to obtain the probability generating function of the proposed model (2.4).

The Abel series distribution and QNBD.

Charalambides (1990) explored the use of the Abel series and introduced the family of Abel series distributions with applications to fluctuations of sample functions of stochastic processes. Nandi \& Das (1994) defined a family of Abel series distributions for real valued parameters $r$ and $b$ by its probability function: 


\section{QUASI-NEGATIVE-BINOMIAL DISTRIBUTION PROPERTIES}

$$
\begin{gathered}
P(x)=\frac{r(r+b x)^{x-1} h(x, b)}{f(r)} \\
x=0,1,2, \ldots \ldots \ldots
\end{gathered}
$$

where $h(x, b) \geq 0 ; \quad r \geq 0 \quad$ if $\quad b \geq 0$ and $r+x b \geq 0$ if $b \leq 0 ; f(r)$ is finite and positive function given by:

$$
f(r)=\sum_{x=0}^{\infty} r(r+b x)^{x-1} h(x, b)
$$

and

$$
h(x, b)=\left.\frac{1}{x !} \frac{d^{x} f(r)}{d r^{x}}\right|_{r=-x b} .
$$

Taking

$$
f(r)=(c-r)^{-a}
$$

results in

$$
h(x, b)=\frac{(a+x-1) !}{(a-1) ! x !}(c+b x)^{-a-x}
$$

and, using (3.1), gives

$$
\begin{gathered}
P(X: x)=\left(\begin{array}{c}
a+x-1 \\
x
\end{array}\right) \frac{r(r+b x)^{x-1}(c+b x)^{-a-x}}{(c-r)^{-a}} \\
x=0,1, \ldots \ldots .
\end{gathered}
$$

Finally, taking

and

$$
\frac{r}{(c-r)}=\theta_{1}, \frac{b}{(c-r)}=\theta_{2}
$$

$$
\frac{c}{(c-r)}=1+\frac{r}{(c-r)}=1+\theta_{1}
$$

the quasi-negative-binomial distribution (2.4) is obtained. Hence, the QNBD is a member of the Abel series of distributions.

Structural properties.

Some of the structural properties that describe the nature of the quasi-negativebinomial distribution were studied. These properties are described as follows:
Convolution property.

Using (3.2) it is possible to show that quasi-negative-binomial variates possess the important - and very desirable - convolution property given by Theorem 4.1: The sum of two independent quasi-negative-binomial variates $X_{1}$ and $X_{2}$ with parameters $\left(a_{1}, \theta_{1}, \theta_{2}\right)$ and $\left(a_{2}, \theta_{1}, \theta_{2}\right)$, respectively, is a quasi-negativebinomial variate with parameters $\left(a_{1}+a_{2}, \theta_{1}, \theta_{2}\right)$.

Proof:

The sum of the probabilities of the QNBD equals unity, therefore from (3.2) the following results:

$$
(c-r)^{-a}=\sum_{x=0}^{\infty}\left(\begin{array}{c}
a+x-1 \\
x
\end{array}\right) r(r+b x)^{x-1}(c+b x)^{-a-x}
$$

Considering the expansion of $(c-r)^{-\left(a_{1}+a_{2}\right)}=(c-r)^{-a_{1}}(c-r)^{-a_{2}}$ as a single series of Abel polynomials on the lefthand side and the product of two series of Abel polynomials on the right-hand side, using (4.1) and simplifying, the following identity is obtained:

$$
\begin{array}{r}
\left(\begin{array}{c}
a_{1}+a_{2}+x-1 \\
x
\end{array}\right) r(r+b x)^{x-1}(c+b x)^{-\left(a_{1}+a_{2}+x\right)} \\
=\sum_{t=0}^{x}\left(\begin{array}{c}
a_{1}+t-1 \\
t
\end{array}\right)\left(\begin{array}{c}
a_{2}+(x-t)-1 \\
x-t
\end{array}\right) r(r+b t)^{t-1} r\left(r+b(x-t)^{x-t-1}\right. \\
(c+b t)^{-\left(a_{1}+t\right)}\left(c+b(x-t)^{-\left(a_{2}+x-t\right)}\right.
\end{array}
$$

This identity reduces to a Vandermonde-type identity on $b=0$, Lagrangian Probability Distribution (Consul \& Famoye, 2006).

Assuming the sum $X_{1}+X_{2}=x$, then by definition:

$$
P\left(\left(X_{1}+X_{2}\right): x\right)=\sum_{t=0}^{x} P_{t}\left(a_{1}, r, b, c\right) P_{x-t}\left(a_{2}, r, b, c\right)
$$




$$
\begin{aligned}
& =\frac{1}{(c-r)^{-\left(a_{1}+a_{2}\right)}} \sum_{t=0}^{x}\left(\begin{array}{c}
a_{1}+t-1 \\
t
\end{array}\right)\left(\begin{array}{c}
a_{2}+(x-t)-1 \\
x-t
\end{array}\right) r(r+b x)^{t-1} \\
& r\left(r+b(x-t)^{x-t-1}(c+b t)^{-\left(a_{1}+t\right)}\left(c+b(x-t)^{-\left(a_{2}+x-t\right)}\right.\right.
\end{aligned}
$$

Using the result (4.2) in the above gives:

$$
\begin{aligned}
& P\left(\left(X_{1}+X_{2}\right): x\right) \\
= & \left(\begin{array}{c}
a_{1}+a_{2}+x-1 \\
x
\end{array}\right) \frac{(r+b x)^{x-1}(c+b x)^{-\left(a_{1}+a_{2}+x\right)}}{(c-r)^{-\left(a_{1}+a_{2}\right)}} .
\end{aligned}
$$

Next, taking

$$
\begin{gathered}
\frac{r}{(c-r)}=\theta_{1}, \frac{b}{(c-r)}=\theta_{2} \quad \text { and } \\
\frac{c}{(c-r)}=1+\frac{r}{(c-r)}=1+\theta_{1}
\end{gathered}
$$

results in the convolution property:

$$
\begin{aligned}
P\left(X_{1}+X_{2}\right. & =x) \\
& =\left(\begin{array}{c}
a_{1}+a_{2}+x-1 \\
x
\end{array}\right) \theta_{1}\left(\theta_{1}+\theta_{2} x\right)^{x-1} \\
& \left(1+\theta_{1}+\theta_{2} x\right)^{-\left(a_{1}+a_{2}+x\right)} \\
& =P_{X}\left(a_{1}+a_{2}, \theta_{1}, \theta_{2}\right)
\end{aligned}
$$

More generally the sum of $\mathrm{n}$ independent quasinegative-binomial variates with parameters $\left(a_{i}, \theta_{1}, \theta_{2}\right), i=1,2, \ldots \ldots . . n$ is also a quasinegative-binomial variate with parameters $\left(\sum a_{i}, \theta_{1}, \theta_{2}\right)$.

\section{Unimodality}

The QNBD is unimodal according to the Lemma: if the mixing distribution is nonnegative, continuous, and unimodal then the resulting distribution is unimodal. (Holgate, 1970) Thus, the proposed model is unimodal since the mixing distribution is the gamma distribution, which is unimodal.

Theorem 4.2: The QNB model (2.4) is unimodal for all values of $\left(a, \theta_{1}, \theta_{2}\right)$ and the mode is $x=0$ if $a \theta_{1}<1$ and, for $a \theta_{1}>1$, the mode is at some point $x=M$ such that

$$
\frac{a \theta_{1}-1}{1-\theta_{1}}<M<\frac{\theta_{1}(a-1)}{1-\theta_{1}} .
$$

Proof:

The QNBD model (2.4) gives the ratio

$$
\begin{aligned}
& \frac{P(1)}{P(0)}=\frac{a \theta_{1}\left(1+\theta_{1}\right)^{a}}{\left(1+\theta_{1}+\theta_{2}\right)^{a+1}}<1 \text { If } a \theta_{1}<1, \\
& \text { since } \frac{\left(1+\theta_{1}\right)^{a}}{\left(1+\theta_{1}+\theta_{2}\right)^{a+1}}<1 \quad \forall\left(a, \theta_{1}, \theta_{2}\right)
\end{aligned}
$$

In general, the ratio of any two successive probabilities of QNBD (2.4) is:

$$
\begin{aligned}
& \frac{P(x+1)}{P(x)} \\
= & \frac{a+x}{x+1} \frac{\left(\theta_{1}+\theta_{2}+x \theta_{2}\right)^{x}}{\left(\theta_{1}+x \theta_{2}\right)^{x-1}} \frac{\left(1+\theta_{1}+x \theta_{2}\right)^{a+x}}{\left(1+\theta_{1}+\theta_{2}+x \theta_{2}\right)^{a+x+1}}
\end{aligned}
$$

Since $\frac{\left(1+\theta_{1}+x \theta_{2}\right)^{a+x}}{\left(1+\theta_{1}+\theta_{2}+x \theta_{2}\right)^{a+x+1}}<1$

$\forall\left(a, \theta_{1}, \theta_{2}\right)$, the ratio $\frac{P(x+1)}{P(x)}<1$, if $\frac{\theta_{1}(a+x)}{x+1}<\frac{\left(1+x \theta_{2} \theta_{1}^{-1}\right)^{x-1}}{\left(1+\theta_{2} \theta_{1}^{-1}+x \theta_{2} \theta_{1}^{-1}\right)^{x}}$, which is true only if $a \theta_{1}<1$ as $\frac{\left(1+x \theta_{2} \theta_{1}^{-1}\right)^{x-1}}{\left(1+\theta_{2} \theta_{1}^{-1}+x \theta_{2} \theta_{1}^{-1}\right)^{x}}<1 \quad \forall\left(\theta_{1}, \theta_{2}\right)$. Hence, for $a \theta_{1}<1$, the ratio $\frac{P(x+1)}{P(x)}$ is a nonincreasing function, therefore the mode of the distribution is $x=0$. Suppose $a \theta_{1}>1$ and the mode is at $x=M$, the ratio defined by (4.3) gives two inequalities: 


$$
\begin{aligned}
\frac{P(M+1)}{P(M)}= & \\
& \frac{a+M}{M+1} \frac{\left(\theta_{1}+\theta_{2}+M \theta_{2}\right)^{M}}{\left(\theta_{1}+M \theta_{2}\right)^{M-1}} \\
& \frac{\left(1+\theta_{1}+M \theta_{2}\right)^{a+M}}{\left(1+\theta_{1}+\theta_{2}+M \theta_{2}\right)^{a+M+1}} \\
& <1
\end{aligned}
$$

and

$$
\begin{aligned}
\frac{P(M)}{P(M-1)}= & \\
& \frac{a+M-1}{M} \frac{\left(\theta_{1}+M \theta_{2}\right)^{M-1}}{\left(\theta_{1}-\theta_{2}+M \theta_{2}\right)^{M-2}} \\
& \frac{\left(1+\theta_{1}-\theta_{2}+M \theta_{2}\right)^{a+M-1}}{\left(1+\theta_{1}+M \theta_{2}\right)^{a+M}} \\
& >1
\end{aligned}
$$

By inequality (4.4):

$$
\frac{\theta_{1}(a+M)}{M+1}<\frac{\left(1+M \theta_{2} \theta_{1}^{-1}\right)^{M-1}}{\left(1+\theta_{2} \theta_{1}^{-1}+M \theta_{2} \theta_{1}^{-1}\right)^{M}}<1
$$

since

$$
\frac{\left(1+M \theta_{2} \theta_{1}^{-1}\right)^{M-1}}{\left(1+\theta_{2} \theta_{1}^{-1}+M \theta_{2} \theta_{1}^{-1}\right)^{M}}<1 \quad \forall\left(\theta_{1}, \theta_{2}\right)
$$

The inequality (4.6) gives the lower bond to $M$ as:

$$
M>\frac{a \theta_{1}-1}{1-\theta_{1}} \text {. }
$$

And by inequality (4.5):

$$
\begin{aligned}
& \frac{a+M-1}{M} \frac{\left(\theta_{1}+M \theta_{2}\right)^{M-1}}{\left(\theta_{1}-\theta_{2}+M \theta_{2}\right)^{M-2}} \\
& >\frac{\left(1+\theta_{1}+M \theta_{2}\right)^{a+M}}{\left(1+\theta_{1}-\theta_{2}+M \theta_{2}\right)^{a+M-1}}
\end{aligned}
$$

Again

$$
\frac{\left(1+\theta_{1}+M \theta_{2}\right)^{a+M}}{\left(1+\theta_{1}-\theta_{2}+M \theta_{2}\right)^{a+M-1}}>1 \quad \forall\left(a, \theta_{1}, \theta_{2}\right)
$$

gives

$$
\frac{\theta_{1}(a+M-1)}{M}>\frac{\left(1-\theta_{2} \theta_{1}^{-1}+M \theta_{2} \theta_{1}^{-1}\right)^{M-2}}{\left(1+M \theta_{2} \theta_{1}^{-1}\right)^{M-1}}
$$

because

$$
a \theta_{1}>1, \frac{\theta_{1}(a+M-1)}{M}>1 \forall\left(\theta_{1}, \theta_{2}\right)
$$

and

$$
\frac{\left(1-\theta_{2} \theta_{1}^{-1}+M \theta_{2} \theta_{1}^{-1}\right)^{M-2}}{\left(1+M \theta_{2} \theta_{1}^{-1}\right)^{M-1}}<1 \quad \forall\left(\theta_{1}, \theta_{2}\right)
$$

Thus, (4.8) can be written as:

$$
\frac{\theta_{1}(a+M-1)}{M}>1>\frac{\left(1-\theta_{2} \theta_{1}^{-1}+M \theta_{2} \theta_{1}^{-1}\right)^{M-2}}{\left(1+M \theta_{2} \theta_{1}^{-1}\right)^{M-1}}
$$

which gives the upper bond to $M$ as:

$$
M<\frac{\theta_{1}(a-1)}{1-\theta_{1}}
$$

By combining (4.7) and (4.9):

$$
\frac{a \theta_{1}-1}{1-\theta_{1}}<M<\frac{\theta_{1}(a-1)}{1-\theta_{1}}
$$

the proof is completed.

Probability generating function

Consul \& Shenton $(1972,1974)$ showed that the derivation of the probability generating function (PGF) of a generalized Poisson variate is not straightforward and is based on the power series expansion of a function in terms of another variable (see GPD by Consul-1989). As they show, the PGF of a generalized Poisson variate $(\theta, \alpha \theta)$ is:

$$
G_{x}(u)=E\left(u^{X}\right)=e^{\theta(t-1)}
$$


where $t=u e^{\alpha \theta(t-1)}, \quad$ and $u$ is a dummy variable.

Similar to the generalized Poisson variate, the PGF of QNBD also does not seem to be straightforward. Therefore, by compounding the restricted generalized Poisson model $(\theta, \alpha \theta)$ with the gamma distribution $\gamma(a, b)$, where $\theta_{1}=b^{-1}$ and $\theta_{2}=\alpha b^{-1}$, and using a theorem by Feller (1943), the PGF of a QNBD is:

$$
\begin{aligned}
& G_{x}(u)=\frac{b^{a}}{\Gamma(a)} \int_{0}^{\infty} e^{-b \theta} \theta^{a-1} e^{\theta(t-1)} d \theta \\
& =\left(1+\theta_{1} b-\theta_{1} t\right)^{-a}, \theta_{1}=b^{-1} \text { and } \theta_{2}=\alpha b^{-1}
\end{aligned}
$$

where $t=u e^{\alpha \theta(t-1)}$

The function $t(u)$ can be written explicitly using Lagrange's Theorem (see Whittaker and Watson, 1927) as:

$$
t=\sum_{n=1}^{\infty} e^{-n \alpha \theta} \frac{(n \alpha \theta)^{n-1}}{n !} u^{n}
$$

Since $\theta$ is varying as gamma distribution $\theta \sim \gamma(a, b)$, the equation above gives:

$t=\sum_{n=1}^{\infty} u^{n} \frac{(n \alpha)^{n-1}}{n !} \frac{b^{a}}{\Gamma(a)} \int_{0}^{\infty} e^{-\theta(b+n \alpha)} \theta^{a+n-2} d \theta$

And, after simplification results in:

$$
t=\sum_{n=1}^{\infty} u^{n} \frac{\Gamma(a+n-1)}{\Gamma(a) n !} \frac{b^{a}(n \alpha)^{n-1}}{(b+n \alpha)^{a+n-1}}
$$

Taking $\theta_{1}=b^{-1}$ and $\theta_{2}=\alpha b^{-1}$ :

$$
t=\sum_{n=1}^{\infty} u^{n}\left(\begin{array}{c}
a+n-2 \\
n-1
\end{array}\right) \frac{n^{n-2} \theta_{2}^{n-1}}{\left(1+n \theta_{2}\right)^{a+n-1}}
$$

Hence the PGF of a QNBD (2.4) is:

$$
G_{x}(u)=\left(1+\theta_{1} b-\theta_{1} t\right)^{-a}
$$

where

$$
t=\sum_{n=1}^{\infty} u^{n}\left(\begin{array}{c}
a+n-2 \\
n-1
\end{array}\right) \frac{n^{n-2} \theta_{2}^{n-1}}{\left(1+n \theta_{2}\right)^{a+n-1}} .
$$

Recurrence relation between the moments

Suppose $\mu_{k}^{\prime}\left(a, \theta_{1}\right)$ denotes the $\mathrm{r}^{\text {th }}$ moment about the origin of a QNBD (2.4), then

$$
\begin{aligned}
& \mu_{k}^{\prime}\left(a, \theta_{1}\right)=\theta_{1} \sum_{x=0}^{\infty} x^{k} \frac{(a+x-1) !}{(a-1) ! x !} \frac{\theta_{1}\left(\theta_{1}+\theta_{2} x\right)^{x-1}}{\left(1+\theta_{1}+\theta_{2} x\right)^{a+x}} \\
& =\theta_{1} \sum_{x=1}^{\infty} x^{k-1} \frac{(a+x-1) !}{(a-1) !(x-1) !} \frac{\theta_{1}\left(\theta_{1}+\theta_{2} x\right)^{x-1}}{\left(1+\theta_{1}+\theta_{2} x\right)^{a+x}}
\end{aligned}
$$

taking $x=x+1$ and expanding $(x+1)^{k-1}$ results in:

$$
\begin{aligned}
\mu_{k}^{\prime}\left(a, \theta_{1}\right)= & \\
& a \theta_{1} \sum_{j=0}^{k-1}\left(\begin{array}{c}
k-1 \\
j
\end{array}\right) \sum_{x=0}^{\infty} x^{j}\left(\overline{\theta_{1}+\theta_{2}}+\theta_{2} x\right) \\
& \frac{(\overline{a+1}+x-1) !}{(\overline{a+1}-1) ! x !} \frac{\theta_{1}\left(\overline{\theta_{1}+\theta_{2}}+\theta_{2} x\right)^{x-1}}{\left(1+\overline{\theta_{1}+\theta_{2}}+\theta_{2} x\right)^{\overline{a+1}+x}}
\end{aligned}
$$

Converting the above series into $\mu_{k}^{\prime}\left(a, \theta_{1}\right)$ functions the recurrence relation

$$
\begin{aligned}
\mu_{k}^{\prime}\left(a, \theta_{1}\right)= & a \theta_{1} \sum_{j=0}^{k-1}\left(\begin{array}{c}
k-1 \\
j
\end{array}\right) \\
& {\left[\begin{array}{l}
\mu_{j}^{\prime}\left(a+1, \theta_{1}+\theta_{2}\right)+ \\
\frac{\theta_{2}}{\left(\theta_{1}+\theta_{2}\right)} \mu_{j+1}^{\prime}\left(a+1, \theta_{1}+\theta_{2}\right)
\end{array}\right] }
\end{aligned}
$$

is obtained.

Where $\mu_{j}^{\prime}\left(a+1, \theta_{1}+\theta_{2}\right)$ is the $\mathrm{j}^{\text {th }}$ moment about the origin of a QNBD with parameters $\left(a+1, \theta_{1}+\theta_{2}, \theta_{2}\right)$. The relation (4.10) is used to determine the moments about the origin of a QNBD. Thus the mean of the distribution is:

$$
\mu_{1}^{\prime}=a \theta_{1}\left[1+\frac{\theta_{2}}{\left(\theta_{1}+\theta_{2}\right)} \mu_{1}^{\prime}\left(a+1, \theta_{1}+\theta_{2}\right)\right]
$$




\section{QUASI-NEGATIVE-BINOMIAL DISTRIBUTION PROPERTIES}

Using (4.10) recursively on the function $\mu_{1}^{\prime}$, the mean is $\mu_{1}^{\prime}=a \theta_{12} F_{0}\left[1, a+1, ; \theta_{2}\right]$, where ${ }_{2} F_{0}\left[1, a+1, \ldots \theta_{2}\right]$ is a hypergeometric function defined by:

$$
{ }_{2} F_{0}\left[1, a+1, \ldots ; \theta_{2}\right]=\sum_{j=0}^{\infty} 1^{[j]}(a+1)^{[j]} \frac{\theta_{2}^{j}}{j !} .
$$

The second moment about the origin is determined from (4.10) as:

$$
\mu_{2}^{\prime}=a \theta_{1}\left[\begin{array}{l}
1+\frac{\theta_{1}+2 \theta_{2}}{\theta_{1}+\theta_{2}} \mu_{1}^{\prime}\left(a+1, \theta_{1}+\theta_{2}\right)+ \\
\frac{\theta_{2}}{\theta_{1}+\theta_{2}} \mu_{2}^{\prime}\left(a+1, \theta_{1}+\theta_{2}\right)
\end{array}\right]
$$

Repeated use of (4.12) on the function $\mu_{2}^{\prime}$ gives:

$$
\mu_{2}^{\prime}=a \theta_{1}\left\{{ }_{2} F_{0}\left[1, a+1, \ldots ; \theta_{2}\right]+A_{1}\right\}
$$

where

$$
\begin{aligned}
A_{1}= & \frac{\theta_{1}+2 \theta_{2}}{\theta_{1}+\theta_{2}} \mu_{1}^{\prime}\left(a+1, \theta_{1}+\theta_{2}\right)+(a+1) \\
& \theta_{2} \frac{\theta+3 \theta_{2}}{\theta_{1}+2 \theta_{2}} \mu_{1}^{\prime}\left(a+2, \theta_{1}+2 \theta_{2}\right) \\
& +(a+1)(a+2) \theta_{2}^{2} \frac{\theta_{1}+4 \theta_{2}}{\theta_{1}+3 \theta_{2}} \\
& \mu_{1}^{\prime}\left(a+3, \theta_{1}+3 \theta_{2}\right)+\ldots
\end{aligned}
$$

Repeated use of (4.11) on the function $\mu_{1}^{\prime}$ gives:

$$
\begin{gathered}
A_{1}=\left(\theta_{1}+2 \theta_{2}\right)(a+1)_{2} F_{0}\left[2, a+2,{ }_{-} ; \theta_{2}\right]+\theta_{2}^{2} \\
(a+1)(a+2)_{2} F_{0}\left[3, a+3, \theta_{2}\right]
\end{gathered}
$$

On substituting the value of $A_{1}$ in (4.12) the second moment is obtained by:

$$
\begin{aligned}
\mu_{2}^{\prime}= & a \theta_{12} F_{0}\left[1, a+1, ; \theta_{2}\right] \\
& +\theta_{1}\left(\theta_{1}+2 \theta_{2}\right) a(a+1){ }_{2} F_{0}\left[2, a+2, ; \theta_{2}\right] \\
& +\theta_{1} \theta_{2}{ }^{2} a(a+1)(a+2){ }_{2} F_{0}\left[3, a+3, \ldots ; \theta_{2}\right]
\end{aligned}
$$

Placing $k=3$ in (4.10) the third moment is obtained by:

$$
\begin{aligned}
\mu_{3}^{\prime}= & a \theta_{1}\left[1+\frac{2 \theta_{1}+3 \theta_{2}}{\theta_{1}+\theta_{2}} \mu_{1}^{\prime}\left(a+1, \theta_{1}+\theta_{2}\right)\right. \\
& +\frac{\theta_{1}+3 \theta_{2}}{\theta_{1}+\theta_{2}} \mu_{2}^{\prime}\left(a+1, \theta_{1}+\theta_{2}\right) \\
& \left.+\frac{\theta_{2}}{\theta_{1}+\theta_{2}} \mu_{3}^{\prime}\left(a+1, \theta_{1}+\theta_{2}\right)\right]
\end{aligned}
$$

Repeated use of (4.14) on the function $\mu_{3}^{\prime}$ gives: $\mu_{3}^{\prime}=a \theta_{1}\left\{{ }_{2} F_{0}\left[1, a+1, \ldots ; \theta_{2}\right]+A_{2}+A_{3}\right\}$ (4.15) where

$$
A_{2}=\frac{2 \theta_{1}+3 \theta_{2}}{\theta_{1}+\theta_{2}} \mu_{1}^{\prime}\left(a+1, \theta_{1}+\theta_{2}\right)+(a+1) \theta_{2}
$$$$
\frac{2 \theta_{1}+5 \theta_{2}}{\theta_{1}+2 \theta_{2}} \mu_{1}^{\prime}\left(a+2, \theta_{1}+2 \theta_{2}\right)
$$$$
+(a+1)(a+2) \theta_{2}^{2}
$$

$$
\frac{2 \theta_{1}+7 \theta_{2}}{\theta_{1}+3 \theta_{2}} \mu_{1}^{\prime}\left(a+3, \theta_{1}+3 \theta_{2}\right)+\ldots
$$

and

$$
\begin{aligned}
& A_{3}=\frac{\theta_{1}+3 \theta_{2}}{\theta_{1}+\theta_{2}} \mu_{2}^{\prime}\left(a+1, \theta_{1}+\theta_{2}\right)+(a+1) \theta_{2} \\
& \frac{\theta_{1}+4 \theta_{2}}{\theta_{1}+2 \theta_{2}} \mu_{2}^{\prime}\left(a+2, \theta_{1}+2 \theta_{2}\right) \\
& +(a+1)(a+2) \theta_{2}^{2} \\
& \frac{\theta_{1}+5 \theta_{2}}{\theta_{1}+3 \theta_{2}} \mu_{2}^{\prime}\left(a+3, \theta_{1}+3 \theta_{2}\right)+\ldots
\end{aligned}
$$

Repeated use of (4.11) in (4.16) gives:

$$
\begin{aligned}
A_{2}= & \left(2 \theta_{1}+3 \theta_{2}\right)(a+1)_{2} F_{0}\left[2, a+2,{ }_{-} ; \theta_{2}\right]+2 \theta_{2}^{2} \\
& (a+1)(a+2){ }_{2} F_{0}\left[3, a+3,{ }_{-} ; \theta_{2}\right]
\end{aligned}
$$

Converting $\mu_{2}^{\prime}$ functions on the right hand side of (4.17) into $\mu_{1}^{\prime}$ functions by the repeated use of (4.12) and using (4.11) on the function $\mu_{1}^{\prime}$ gives: 
HASSAN \& BILAL

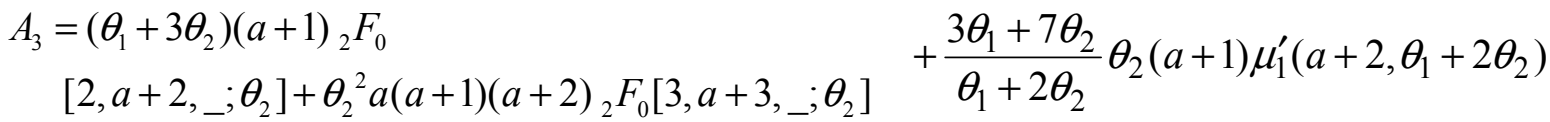

$$
\begin{aligned}
& +\left(\theta_{1}^{2}+6 \theta_{1} \theta_{2}+9 \theta_{2}^{2}\right)(a+1)(a+2)_{2} F_{0}\left[3, a+3,{ }_{-} ; \theta_{2}\right] \\
& +\left(3 \theta_{1} \theta_{2}+10 \theta_{2}^{2}\right)(a+1)(a+2)(a+3)_{2} F_{0}\left[4, a+4, ; \theta_{2}+\frac{3 \theta_{1}+10 \theta_{2}}{\theta_{1}+3 \theta_{2}} \theta_{2}^{2}(a+1)(a+2) \mu_{1}^{\prime}\left(a+3, \theta_{1}+3 \theta_{2}\right)+\ldots\right\} \\
& +3 \theta_{2}^{4}(a+1)(a+2)(a+3)(a+4){ }_{2} F_{0}\left[5, a+5, \ldots ; \theta_{2}\right]
\end{aligned}
$$

Substituting the values of $A_{2}$ and $A_{3}$ into (4.15)

results in:

$$
\begin{aligned}
\mu_{3}^{\prime}= & a \theta_{12} F_{0}\left[1, a+1,{ }_{2} ; \theta_{2}\right]+\theta_{1} \\
& 3\left(\theta_{1}+2 \theta_{2}\right) a(a+1){ }_{2} F_{0}\left[2, a+2, \theta_{2}\right] \\
& +\theta_{1}\left(\theta_{1}^{2}+6 \theta_{1} \theta_{2}+12 \theta_{2}^{2}\right) a(a+1) \\
& (a+2)_{2} F_{0}\left[3, a+3,{ }_{-} ; \theta_{2}\right] \\
& +\theta_{1} \theta_{2}\left(3 \theta_{1} \theta_{2}+10 \theta_{2}^{2}\right) a(a+1) \\
& (a+2)(a+3)_{2} F_{0}\left[4, a+4,{ }_{-} ; \theta_{2}\right] \\
& +3 \theta_{1} \theta_{2}^{4} a(a+1)(a+2)(a+3) \\
& (a+4)_{2} F_{0}\left[5, a+5,{ }_{-} ; \theta_{2}\right]
\end{aligned}
$$

Similarly the fourth moment can be determined from (4.10) as:

$$
\begin{aligned}
\mu_{4}^{\prime}= & a \theta_{1}\left[1+\frac{3 \theta_{1}+4 \theta_{2}}{\theta_{1}+\theta_{2}} \mu_{1}^{\prime}\left(a+1, \theta_{1}+\theta_{2}\right)\right. \\
& +\frac{3\left(\theta_{1}+2 \theta_{2}\right)}{\theta_{1}+\theta_{2}} \mu_{2}^{\prime}\left(a+1, \theta_{1}+\theta_{2}\right) \\
& +\frac{\theta_{1}+4 \theta_{2}}{\theta_{1}+\theta_{2}} \mu_{3}^{\prime}\left(a+1, \theta_{1}+\theta_{2}\right) \\
& \left.+\frac{\theta_{2}}{\theta_{1}+\theta_{2}} \mu_{4}^{\prime}\left(a+1, \theta_{1}+\theta_{2}\right)\right]
\end{aligned}
$$

Repeated use of (4.18) on the function $\mu_{4}^{\prime}$ gives:

$$
\begin{aligned}
& \mu_{4}^{\prime}=a \theta_{1}\left[{ }_{2} F_{0}\left[1, a+1 ;_{-}, \theta_{2}\right]+\right. \\
& \left\{\frac{3 \theta_{1}+4 \theta_{2}}{\theta_{1}+\theta_{2}} \mu_{1}^{\prime}\left(a+1, \theta_{1}+\theta_{2}\right)\right.
\end{aligned}
$$

$$
\begin{aligned}
& +3\left\{\frac{\theta_{1}+2 \theta_{2}}{\theta_{1}+\theta_{2}} \mu_{2}^{\prime}\left(a+1, \theta_{1}+\theta_{2}\right)\right. \\
& +\frac{\theta_{1}+3 \theta_{2}}{\theta_{1}+2 \theta_{2}} \theta_{2}(a+1) \mu_{2}^{\prime}\left(a+2, \theta_{1}+2 \theta_{2}\right) \\
& \left.+\frac{\theta_{1}+4 \theta_{2}}{\theta_{1}+3 \theta_{2}} \theta_{2}^{2}(a+1)(a+2) \mu_{2}^{\prime}\left(a+3, \theta_{1}+3 \theta_{2}\right)+\ldots\right\} \\
& +\left\{\frac{\theta_{1}+4 \theta_{2}}{\theta_{1}+\theta_{2}} \mu_{3}^{\prime}\left(a+1, \theta_{1}+\theta_{2}\right)\right. \\
& +\frac{\theta_{1}+5 \theta_{2}}{\theta_{1}+2 \theta_{2}} \theta_{2}(a+1) \mu_{3}^{\prime}\left(a+2, \theta_{1}+2 \theta_{2}\right) \\
& \left.\left.+\frac{\theta_{1}+6 \theta_{2}}{\theta_{1}+3 \theta_{2}} \theta_{2}^{2}(a+1)(a+2) \mu_{3}^{\prime}\left(a+3, \theta_{1}+3 \theta_{2}\right)+\ldots\right\}\right]
\end{aligned}
$$

Repeated use of (4.11), (4.12), and (4.14) recursively on the functions $\mu_{1}^{\prime}, \mu_{2}^{\prime}$ and $\mu_{3}^{\prime}$ respectively with simplifications results in:

$$
\begin{aligned}
& \mu_{4}^{\prime}=a \theta_{12} F_{0}\left[1, a+1,{ }_{2} ; \theta_{2}\right]+\theta_{1} \\
& \left(7 \theta_{1}+14 \theta_{2}\right) a(a+1){ }_{2} F_{0}\left[2, a+2,{ }_{2} ; \theta_{2}\right] \\
& +\theta_{1}\left(6 \theta_{1}^{2}+36 \theta_{1} \theta_{2}+6 \theta_{2}+55 \theta_{2}^{2}\right) a(a+1) \\
& (a+2)_{2} F_{0}\left[3, a+3,{ }_{-} ; \theta_{2}\right] \\
& +\theta_{1}\left(\theta_{1}^{3}+3 \theta_{1} \theta_{2}+12 \theta_{1}^{2} \theta_{2}+63 \theta_{1} \theta_{2}^{2}\right. \\
& \left.+13 \theta_{2}^{2}+114 \theta_{2}^{3}\right) a(a+1)(a+2)(a+3) \\
& { }_{2} F_{0}\left[4, a+4,{ }_{-} ; \theta_{2}\right]+\theta_{1} \theta_{2}\left(6 \theta_{1}^{2} \theta_{2}\right. \\
& \left.+52 \theta_{1} \theta_{2}^{2}+131 \theta_{2}^{3}\right) a(a+1)(a+2) \\
& (a+3)(a+4)_{2} F_{0}\left[5, a+5, \ldots ; \theta_{2}\right]+\theta_{1} \theta_{2}^{2} \\
& \left(15 \theta_{1} \theta_{2}^{2}+70 \theta_{2}^{3}\right) a(a+1)(a+2)(a+3) \\
& (a+4)(a+5)_{2} F_{0}\left[6, a+6, \theta_{2} ; \theta_{2}\right]+15 \theta_{1} \theta_{2}^{6} \\
& a(a+1)(a+2)(a+3)(a+4) \\
& (a+5)(a+6)_{2} F_{0}\left[7, a+7, \theta_{-}\right]
\end{aligned}
$$




\section{QUASI-NEGATIVE-BINOMIAL DISTRIBUTION PROPERTIES}

The moments about the origin can be easily verified for the negative-binomial distribution when $\theta_{2}=0$. Further, central moments can be obtained from the moments about origin, thus resulting in the variance:

$$
\begin{aligned}
\mu_{2}= & a \theta_{12} F_{0}\left[1, a+1,{ }_{2} ; \theta_{2}\right]+\theta_{1}\left(\theta_{1}+2 \theta_{2}\right) \\
& a(a+1)_{2} F_{0}\left[2, a+2,{ }_{2} ; \theta_{2}\right] \\
& +\theta_{1} \theta_{2}^{2} a(a+1)(a+2){ }_{2} F_{0} \\
& {\left[3, a+3, \ldots ; \theta_{2}\right]-\left[a \theta_{12} F_{0}\left[1, a+1, \ldots ; \theta_{2}\right]\right]^{2} }
\end{aligned}
$$

The third and fourth central moments are coming in messy forms and are not shown here.

Relation with other distributions.

Theorem 5.1: Let $X=$ a quasi-negativebinomial variate with parameters $\left(a, \theta_{1}, \theta_{2}\right)$. If $a \rightarrow \infty$ such that $a \theta_{1}=\alpha$ and $a \theta_{2}=\lambda$ show that $\mathrm{X}$ tends to generalized Poisson distribution with parameters $(\alpha, \lambda)$.

Proof:

The QNBD can be expressed as:

$$
\begin{gathered}
P(X: x)=\frac{a(a+1) \ldots(a+x-1)}{x !} \\
\frac{\theta_{1}\left(\theta_{1}+\theta_{2} x\right)^{x-1}}{\left(1+\theta_{1}+\theta_{2} x\right)^{a+x}} \\
=\frac{\left(1+a^{-1}\right) \ldots \ldots\left(1+(x-1) a^{-1}\right)}{x !} \\
\frac{\left(a \theta_{1}\right)\left(a \theta_{1}+a \theta_{2} x\right)^{x-1}}{1+(a+x)\left(\theta_{1}+\theta_{2} x\right)+\frac{(a+x)(a+x-1)}{2 !}} \\
\left(\theta_{1}+\theta_{2} x\right)^{2}+\ldots\left(\theta_{1}+\theta_{2} x\right)^{a+x}
\end{gathered}
$$

Taking limit $a \rightarrow \infty$, such that $a \theta_{1}=\alpha$ and $a \theta_{2}=\lambda$ results in a generalized Poisson distribution with parameters $(\alpha, \lambda)$ as defined by Consul \& Jain (1973).

Theorem 5.2: Let $\mathrm{X}=$ a quasi-negativebinomial variate with parameters $\left(a, \theta_{1}, \theta_{2}\right)$. If $a \rightarrow \infty$ such that $a \lambda^{-1}=\alpha$, show that X tends to the Borel-Tanner distribution.

Proof:

Stating (5.1) as:

$$
\begin{gathered}
P(X: x)=\frac{a(a+1) \ldots \ldots(a+x-1)}{x !} \\
\frac{r(r+x)^{x-1} \lambda^{a}}{(r+\lambda+x)^{a+x}}
\end{gathered}
$$

where

$$
r=\frac{\theta_{1}}{\theta_{2}} \text { and } \lambda=\frac{1}{\theta_{2}}
$$

Shifting the support of $\mathrm{x}$ from 0 to $\mathrm{r}$, that is, $x=x-r$, results in:

$$
\begin{aligned}
& P(X: x)= \\
& \quad \frac{a(a+1) \ldots \ldots(a+x-r-1)}{(x-r) !} \frac{r x^{x-r-1} \lambda^{a}}{(\lambda+x)^{a+x-r}} \\
& x=r, r+1, r+2, \ldots \\
& =\frac{r x^{x-r-1}}{(x-r) !} \frac{a(a+1) \ldots \ldots(a+x-r-1)}{\lambda^{x-r}\left(1+x \lambda^{-1}\right)^{a+x-r}}
\end{aligned}
$$

Taking the limit $a \rightarrow \infty$ in such a way so $a \lambda^{-1}=\alpha \quad$ results in the Borel-Tanner distribution

$$
\begin{gathered}
P(X: x)=\frac{r}{(x-r) !} x^{x-r-1} e^{-\alpha x} \alpha^{x-r}, \\
x=r, r+1, r+2, \ldots
\end{gathered}
$$

Theorem 5.3: Let $\mathrm{X}=$ a quasi-negativebinomial variate with parameters $\left(a, \theta_{1}, \theta_{2}\right)$. Show that zero-truncated quasi-negativebinomial distribution tends to quasi-logarithmic series distribution as $a \rightarrow 0$.

Proof:

The zero-truncated quasi-negative-binomial distribution is 


$$
\begin{aligned}
P_{1}(x) & =\frac{\Gamma(a+x)}{\Gamma(a) \Gamma(x+1)} \\
& \frac{\theta_{1}\left(\theta_{1}+\theta_{2} x\right)^{x-1}}{\left[1-\left(1+\theta_{1}\right)^{-a}\right]\left(1+\theta_{1}+\theta_{2} x\right)^{a+x}}, \\
x & =1,2, \ldots . .
\end{aligned}
$$

Writing:

$$
\begin{gathered}
\Gamma(a)\left[1-\left(1+\theta_{1}\right)^{-a}\right]= \\
\Gamma(a)\left[\begin{array}{l}
1-\left(1-a \theta_{1}+\frac{a(a+1)}{2 !}\right. \\
\theta_{1}^{2}-\frac{a(a+1)(a+2)}{3 !} \theta_{1}^{3}+\ldots
\end{array}\right] \\
=\Gamma(a+1)\left[\begin{array}{l}
-\left(\theta_{1}-\frac{(a+1)}{2 !} \theta_{1}^{2}+\right. \\
\left.\frac{(a+1)(a+2)}{3 !} \theta_{1}^{3} \ldots . . .\right)
\end{array}\right]
\end{gathered}
$$

Substituting the value from (5.3) into (5.2) and taking limit $a \rightarrow 0$ the quasi-logarithmic series distribution is obtained:

$$
\begin{aligned}
P_{1}(x) & =\frac{\theta_{1}\left(\theta_{1}+\theta_{2} x\right)^{x-1}}{x\left[-\log \left(1-\theta_{1}\right)\right]\left(1+\theta_{1}+\theta_{2} x\right)^{a+x}}, \\
x & =1,2, \ldots
\end{aligned}
$$

Theorem 5.4: If $X_{1}$ and $X_{2}$ are two independent quasi-negative-binomial variates with parameters $\left(n_{1}, \theta_{1}, \theta_{2}\right)$ and $\left(n_{2}, \theta_{1}, \theta_{2}\right)$, respectively, then the conditional probability of $X_{1}$, given $X_{1}+X_{2}=n$, gives a hypergeometric-QNBD.

Proof:

Because $X_{1}$ and $X_{2}$ are two independent quasi-negative-binomial variates, the conditional probability

$$
\begin{aligned}
P\left[X_{1}: x /\left(X_{1}+X_{2}\right): n\right] & = \\
& \frac{P\left(X_{1}=x, X_{2}=n-x\right)}{\sum_{x=0}^{n} P\left(X_{1}=x, X_{2}=n-x\right)}
\end{aligned}
$$

can be written as

$$
\begin{aligned}
& P\left[X_{1}: x /\left(X_{1}+X_{2}\right): n\right] \\
& \left(\begin{array}{c}
n_{1}+x-1 \\
x
\end{array}\right) \frac{\theta_{1}\left(\theta_{1}+x \theta_{2}\right)^{x-1}}{\left(1+\theta_{1}+x \theta_{2}\right)^{n_{1}+x}} \\
& =\frac{\left(\begin{array}{c}
n_{2}+n-x-1 \\
n-x
\end{array}\right) \frac{\theta_{1}\left(\theta_{1}+(n-x) \theta_{2}\right)^{n-x-1}}{\left(1+\theta_{1}+(n-x) \theta_{2}\right)^{n_{2}+n-x}}}{\left(\begin{array}{c}
n_{1}+n_{2}+n-1 \\
n
\end{array}\right) \frac{\theta_{1}\left(\theta_{1}+n \theta_{2}\right)^{n-1}}{\left(1+\theta_{1}+n \theta_{2}\right)^{n_{1}+n_{2}+n}}} \\
& =\frac{\left(\begin{array}{c}
n_{1}+x-1 \\
x
\end{array}\right)\left(\begin{array}{c}
n_{2}+n-x-1 \\
n-x
\end{array}\right)}{\left(\begin{array}{c}
n_{1}+n_{2}+n-1 \\
n
\end{array}\right)} \\
& \theta_{1}\left(\theta_{1}+x \theta_{2}\right)^{x-1} \\
& \frac{\left(\theta_{1}+(n-x) \theta_{2}\right)^{n-x-1}\left(1+\theta_{1}+n \theta_{2}\right)^{n_{1}+n_{2}+n}}{\left(1+\theta_{1}+x \theta_{2}\right)^{n_{1}+x}} \\
& \left(1+\theta_{1}+(n-x) \theta_{2}\right)^{n_{2}+n-x}\left(\theta_{1}+n \theta_{2}\right)^{n-1}
\end{aligned}
$$

Thus resulting in a new distribution, here called the hypergeometric QNBD. This probability distribution reduces to the classical hypergeometric distribution on $\theta_{2}=0$.

Some characterization.

A number of complex distributions can be reduced to the simpler form QNBD as shown in the following theorems.

Theorem: 6.1. If $\mathrm{X}$ is a quasi-inverse Polya variate with parameters $(n, a, b, t)$, and if $b \rightarrow \infty$ such that $a b^{-1}=\lambda_{1}$ and $t b^{-1}=\lambda_{2}$ show that $\mathrm{X}$ approaches to quasi-negativebinomial variate. 
Proof:

If $\mathrm{X}$ is a quasi-inverse Polya variate with parameters $(n, a, b, t)$, then its probability mass function is:

$$
\begin{aligned}
& P(X: x)=\frac{n}{n+x}\left(\begin{array}{c}
n+x \\
x
\end{array}\right) \\
& \quad \frac{a}{a+x t} \frac{(a+x t)^{[x]}(b+x t)^{[n]}}{(a+b+\overline{n+x} t)^{[n+x]}} \\
& x=0,1,2, \ldots .
\end{aligned}
$$

which can be rewritten as:

$$
\begin{aligned}
P(X: x) & =\frac{n(n+1) \ldots(n+x-1)}{x !} \\
& a(a+x t+1) \ldots(a+x t+x-1) \\
& \frac{(b+x t) \ldots(b+x t+n-1)}{(a+b+\overline{n+x} t) \ldots} \\
& (a+b+\overline{n+x} t+n+x-1)
\end{aligned}
$$

Taking limit $b \rightarrow \infty$ such that $a b^{-1}=\lambda_{1}$ and $t b^{-1}=\lambda_{2}$ results in:

$$
P(X: x)=\left(\begin{array}{c}
n+x-1 \\
x
\end{array}\right) \frac{\left(1+\lambda_{1}+(n+x) \lambda_{2}\right)^{-(n+x)}}{\left(1+n \lambda_{2}\right)^{-n} .}
$$

Incorporating $\quad \theta_{1}=\lambda_{1}\left(1+n \lambda_{2}\right)^{-1} \quad$ and $\theta_{2}=\lambda_{2}\left(1+n \lambda_{2}\right)^{-1}$, the QNBD (2.4) is obtained.

Theorem 6.2: If $\mathrm{X}$ is a generalized negative Polya-Eggenberger variate with parameters $(n, \beta, \alpha, \gamma)$, and if $\beta \rightarrow \infty$ such that $n \beta^{-1}=\lambda_{1}$ and $\gamma \beta^{-1}=\lambda_{2}$ show that $\mathrm{X}$ approaches to quasi-negative-binomial variate.

Proof:

The generalized negative Polya-Eggenberger distribution with parameters $(n, \beta, \alpha, \gamma)$ is:

$$
\begin{gathered}
P(X=x)=\frac{n}{(n+\beta x)}\left(\begin{array}{c}
n+\beta x \\
x
\end{array}\right) \frac{a^{[x]} b^{[n+\beta x-x]}}{(a+b)^{[n+\beta x]}}, \\
x=0,1,2, \ldots \ldots .
\end{gathered}
$$

This can be rewritten as:

$$
\begin{aligned}
& P(X: x)= \\
& \frac{n(n+\beta x-1) \ldots(n+\beta x-x+1)}{x !} \\
& \frac{\alpha^{[x]} \gamma^{[n+\beta x-x]}}{(\alpha+\gamma)^{[n+\beta x]}}
\end{aligned}
$$

Writing

$$
\begin{gathered}
\frac{\gamma^{[n+\beta x-x]}}{(\alpha+\gamma)^{[n+\beta x]}}=\frac{\gamma^{[\alpha]} \gamma^{[n+\beta x-x]}}{\gamma^{[n+\beta x-x+\alpha+x]}} \\
=\frac{\gamma^{[\alpha]}}{(\gamma+n+\beta x-x)^{[\alpha+x]}} \\
=\frac{\gamma(\gamma+1) \ldots(\gamma+\alpha-1)}{(\gamma+n+\beta x-x) \ldots} \\
(\gamma+n+\beta x-x+\alpha+x-1)
\end{gathered}
$$

On substituting this value into (6.1) and taking the limit $\beta \rightarrow \infty$, such that $n \beta^{-1}=\lambda_{1}$ and $\gamma \beta^{-1}=\lambda_{2}$ results in:

$$
\begin{gathered}
P(X: x)=\left(\begin{array}{c}
\alpha+x-1 \\
x
\end{array}\right) \frac{\lambda_{1}\left(\lambda_{1}+x\right)^{x-1} \lambda_{2}^{\alpha}}{\left(\lambda_{1}+\lambda_{2}+x\right)^{\alpha+x}} \\
x=0,1, \ldots
\end{gathered}
$$

Taking $\theta_{1}=\lambda_{1} \lambda_{2}^{-1}$ and $\theta_{2}=\lambda_{2}^{-1}$ QNBD (2.4) is obtained.

Theorem 6.3: If $\mathrm{X}$ is a quasi-inverse hypergeometric variate with parameters $(n, a, b$, $\mathrm{t}$ ), and if $b \rightarrow \infty$ such that $a b^{-1}=\lambda_{1}$ and $t b^{-1}=\lambda_{2}$, show that $\mathrm{X}$ approaches to quasinegative-binomial variate.

Proof:

If $\mathrm{X}$ is a quasi-inverse hypergeometric variate with parameters $(n, a, b, t)$ then its probability mass function is: 
$P(X: x)=\frac{n}{n+x} \frac{a}{a+x t} \frac{\left(\begin{array}{c}a+x t \\ x\end{array}\right)\left(\begin{array}{c}b+n t \\ n\end{array}\right)}{\left(\begin{array}{c}a+b+\overline{n+x} t \\ n+x\end{array}\right)}$

$x=0,1,2, \ldots$.

Restating this as

$$
\begin{aligned}
P(X: x) & =\frac{(n+x-1) !}{(n-1) ! x !} \frac{a}{a+x t} \\
& \frac{(a+x t-x+1)^{[x]}(b+n t-n+1)^{[n]}}{(a+b+(n+x) t-(n+x)+1)^{[n+x]}},
\end{aligned}
$$

expanding

$\frac{a}{a+x t} \frac{(a+x t-x+1)^{[x]}(b+n t-n+1)^{[n]}}{(a+b+(n+x) t-(n+x)+1)^{[n+x]}}$

and taking limit $b \rightarrow \infty$, such that $a b^{-1}=\lambda_{1}$ and $t b^{-1}=\lambda_{2}$ the equation reduces to:

$$
\begin{gathered}
P(X: x)=\left(\begin{array}{c}
n+x-1 \\
x
\end{array}\right) \\
\frac{\lambda_{1}\left(\lambda_{1}+x \lambda_{2}\right)^{x-1}\left(1+\lambda_{1}+(n+x) \lambda_{2}\right)^{-(n+x)}}{\left(1+n \lambda_{2}\right)^{-n} .} .
\end{gathered}
$$

Taking $\quad \theta_{1}=\lambda_{1}\left(1+n \lambda_{2}\right)^{-1}$

and

$\theta_{2}=\lambda_{2}\left(1+n \lambda_{2}\right)^{-1}$, the QNBD (2.4) is obtained.

Theorem 6.4: If $X_{1}$ and $X_{2}$ are two independent non-negative integer valued random variables such that

$$
\begin{aligned}
& P\left[X_{1}: 0 /\left(X_{1}+X_{2}\right): x\right]= \\
& \frac{n_{2}^{\left[n_{1}\right]}}{\left(n_{2}+x\right)^{\left[n_{1}\right]}} \frac{\left(1+\theta_{1}+x \theta_{2}\right)^{n_{1}}}{\left(1+\theta_{1}\right)^{n_{1}}}
\end{aligned}
$$

$$
\begin{aligned}
P\left[X_{1}: 1 /\left(X_{1}+X_{2}\right): x\right] & \\
& \frac{x n_{2}^{\left[n_{1}\right]}}{\left(n_{2}+x-1\right)\left(n_{2}+x\right)^{\left[n_{1}\right]}} \\
& \frac{n_{1} \theta_{1}}{\left(1+\theta_{1}+\theta_{2}\right)^{n_{1}+1}} \frac{\left(\theta_{1}+(x-1) \theta_{2}\right)^{x-2}}{\left(\theta_{1}+x \theta_{2}\right)^{x-1}} \\
& \frac{\left(1+\theta_{1}+x \theta_{2}\right)^{n_{1}+n_{2}+x}}{\left(1+\theta_{1}+(x-1) \theta_{2}\right)^{n_{2}+x-1}}
\end{aligned}
$$

where $n_{2}{ }^{\left[n_{1}\right]}=n_{2}\left(n_{2}+1\right) \ldots \ldots\left(n_{2}+n_{1}-1\right)$ and $\theta_{1}>0, \theta_{2}>0, n_{1}>0, n_{2}>0$, show that $X_{1}$ and $X_{2}$ are two independent quasi-negativebinomial variates with parameters $\left(n_{1}, \theta_{1}, \theta_{2}\right)$ and $\left(n_{2}, \theta_{1}, \theta_{2}\right)$ respectively.

Proof:

Let $\quad P\left(X_{1}=x_{1}\right)=f\left(x_{1}\right) \quad$ and $P\left(X_{2}=x_{2}\right)=g\left(x_{2}\right)$. By condition (i)

$\frac{f(0) g(x)}{\sum_{t=0}^{x} f(t) g(x-t)}=\frac{n_{2}{ }^{\left[n_{1}\right]}}{\left(n_{2}+x\right)^{\left[n_{1}\right]}} \frac{\left(1+\theta_{1}+x \theta_{2}\right)^{n_{1}}}{\left(1+\theta_{1}\right)^{n_{1}}}$

and by condition (ii)

$$
\begin{gathered}
\frac{f(1) g(x-1)}{\sum_{t=0}^{x} f(t) g(x-t)}=\frac{x n_{2}^{\left[n_{1}\right]}}{\left(n_{2}+x-1\right)\left(n_{2}+x\right)^{\left[n_{1}\right]}} \\
\frac{n_{1} \theta_{1}}{\left(1+\theta_{1}+\theta_{2}\right)^{n_{1}+1}} \\
\frac{\left(\theta_{1}+(x-1) \theta_{2}\right)^{x-2}}{\left(\theta_{1}+x \theta_{2}\right)^{x-1}} \frac{\left(1+\theta_{1}+x \theta_{2}\right)^{n_{1}+n_{2}+x}}{\left(1+\theta_{1}+(x-1) \theta_{2}\right)^{n_{2}+x-1}}
\end{gathered}
$$

and
Dividing (6.2) by (6.3) results in: 


\section{QUASI-NEGATIVE-BINOMIAL DISTRIBUTION PROPERTIES}

$$
\begin{aligned}
\frac{f(0) g(x)}{f(1) g(x-1)}= & \\
& \frac{\left(n_{2}+x-1\right)}{x} \frac{\left(1+\theta_{1}+\theta_{2}\right)^{n_{1}+1}}{n_{1} \theta_{1}\left(1+\theta_{1}\right)^{n_{1}}} \\
& \frac{\left(\theta_{1}+x \theta_{2}\right)^{x-1}}{\left(\theta_{1}+(x-1) \theta_{2}\right)^{x-2}} \\
& \frac{\left(1+\theta_{1}+(x-1) \theta_{2}\right)^{n_{2}+x-1}}{\left(1+\theta_{1}+x \theta_{2}\right)^{n_{2}+x}}
\end{aligned}
$$

which gives a recurrence relation $g(x)=$

$$
\begin{aligned}
& \frac{f(1)}{f(0)} \frac{\left(1+\theta_{1}+\theta_{2}\right)^{n_{1}+1}}{n_{1} \theta_{1}\left(1+\theta_{1}\right)^{n_{1}}} \\
& \frac{\left(n_{2}+x-1\right)}{x} \frac{\left(\theta_{1}+x \theta_{2}\right)^{x-1}}{\left(\theta_{1}+(x-1) \theta_{2}\right)^{x-2}} \\
& \frac{\left(1+\theta_{1}+(x-1) \theta_{2}\right)^{n_{2}+x-1}}{\left(1+\theta_{1}+x \theta_{2}\right)^{n_{2}+x}} g(x-1)
\end{aligned}
$$

Repeated use of the equation above gives: $g(x)=$

$$
\begin{aligned}
& {\left[\frac{f(1)}{f(0)} \frac{\left(1+\theta_{1}+\theta_{2}\right)^{n_{1}+1}}{n_{1} \theta_{1}\left(1+\theta_{1}\right)^{n_{1}}}\right]^{x}} \\
& \frac{\left(n_{2}+x-1\right) \ldots . n_{2}}{x(x-1) \ldots . .1} \frac{\left(\theta_{1}+x \theta_{2}\right)^{x-1}}{\theta_{1}^{-1}} \\
& \frac{\left(1+\theta_{1}\right)^{n_{2}}}{\left(1+\theta_{1}+x \theta_{2}\right)^{n_{2}+x}} g(0)
\end{aligned}
$$

Substituting $\frac{f(1)}{f(0)}=\frac{n_{1} \theta_{1}\left(1+\theta_{1}\right)^{n_{1}}}{\left(1+\theta_{1}+\theta_{2}\right)^{n_{1}+1}}$ results in:

$$
\begin{aligned}
g(x)= & \left(\begin{array}{c}
n_{2}+x-1 \\
x
\end{array}\right) \frac{\left(n_{2}+x-1\right) \ldots n_{2}}{x(x-1) \ldots \ldots 1} \\
& \frac{\theta_{1}\left(\theta_{1}+x \theta_{2}\right)^{x-1}}{\left(1+\theta_{1}+x \theta_{2}\right)^{n_{2}+x}}\left(1+\theta_{1}\right)^{n_{2}} g(0)
\end{aligned}
$$

$\mathrm{f}$ the above relation represents a probability mass function, then $\sum_{x} g(x)=1 \Rightarrow g(0)=\left(1+\theta_{1}\right)^{-n_{2}}$ and this reduces the equation above to

$$
\begin{gathered}
g(x)=\left(\begin{array}{c}
n_{2}+x-1 \\
x
\end{array}\right) \frac{\left(n_{2}+x-1\right) \ldots n_{2}}{x(x-1) \ldots 1} \\
\frac{\theta_{1}\left(\theta_{1}+x \theta_{2}\right)^{x-1}}{\left(1+\theta_{1}+x \theta_{2}\right)^{n_{2}+x}} \\
x=0,1,2, \ldots
\end{gathered}
$$

This is a quasi-negative-binomial distribution with parameters $\left(n_{2}, \theta_{1}, \theta_{2}\right)$. Similarly it can be shown that $f(x)$ also represents a quasinegative-binomial distribution with parameters $\left(n_{1}, \theta_{1}, \theta_{2}\right)$.

\section{Goodness of Fit}

Due to its complicated likelihood function, the maximum likelihood estimate of the parameters of the proposed distribution are not straightforward and require some iterative procedure such as Fisher's scoring method or the Newton-Rampson method for their solution. Rsoftware provides one such solution. In Rsoftware there exists the function nlm, which minimizes the negative log-likelihood function or equivalently maximizes the log likelihood function for estimating the parameters of the distribution by adopting the Newton-Rampson iterative procedure. A random start procedure is employed, that is, for a set of random starting points, the function $\mathrm{nlm}$ searches recursively until global maxima is reached. To verify that the global maximum has been found the gradient should be equal to zero. The closer the value of the random starting points to the ML estimate, the lesser number of iterations will be required to obtain the global maximum.

Two data sets examine the fitting of the proposed model and compare it with the negative binomial distribution and generalized negative binomial distribution defined by Jain \& Consul (1971). A computer program was developed using R-software to estimate the parameters of the distribution by using the nlm function. The ML estimates of the parameters so obtained are shown at the bottom of the tables. It is evident from tables 4.1 and 4.2 that, in all cases, the Chi-square values of the proposed model give the best fit as compared to other distributions. 
HASSAN \& BILAL

Table 4.1: Absenteeism among shift-workers in steel industry; data from Arbous \& Sichel, 1954

\begin{tabular}{|c|c|c|c|c|}
\hline \multirow[b]{2}{*}{ Count } & \multirow{2}{*}{$\begin{array}{l}\text { Observed } \\
\text { Frequency }\end{array}$} & \multicolumn{3}{|c|}{ Expected Frequencies } \\
\hline & & NBD & $\begin{array}{c}\text { GNBD Jain \& } \\
\text { Consul's (1971) }\end{array}$ & $\begin{array}{l}\text { QNBD Proposed } \\
\text { Model }\end{array}$ \\
\hline 0 & 7 & 12.02 & 10.51 & 10.47 \\
\hline 1 & 16 & 16.16 & 17.45 & 16.05 \\
\hline 2 & 23 & 17.77 & 20.38 & 18.55 \\
\hline 3 & 20 & 18.08 & 20.80 & 19.19 \\
\hline 4 & 23 & 17.65 & 19.88 & 18.72 \\
\hline 5 & 24 & 16.80 & 18.34 & 17.63 \\
\hline 6 & 12 & 15.72 & 16.56 & 16.24 \\
\hline 7 & 13 & 14.52 & 14.78 & 14.74 \\
\hline 8 & 09 & 13.28 & 13.08 & 13.23 \\
\hline 9 & 09 & 12.06 & 11.53 & 11.80 \\
\hline 10 & 08 & 10.89 & 10.13 & 10.46 \\
\hline 11 & 10 & 09.78 & 08.89 & 9.25 \\
\hline 12 & 08 & 08.75 & 07.79 & 8.15 \\
\hline 13 & 07 & 07.80 & 16.83 & 7.18 \\
\hline 14 & 02 & 06.93 & 05.99 & 6.31 \\
\hline 15 & 12 & 06.14 & 05.26 & 5.55 \\
\hline 16 & 03 & 05.43 & 04.61 & 4.88 \\
\hline 17 & 05 & 04.79 & 04.05 & 4.30 \\
\hline 18 & 04 & 04.22 & 03.56 & 3.79 \\
\hline 19 & 02 & 03.17 & 03.14 & 3.34 \\
\hline 20 & 02 & 03.23 & 02.76 & 2.94 \\
\hline 21 & 05 & 02.86 & 02.43 & 2.60 \\
\hline 22 & 05 & 02.50 & 02.15 & 2.30 \\
\hline 23 & 02 & 02.91 & 01.90 & 2.04 \\
\hline 24 & 01 & 01.91 & 01.68 & 1.81 \\
\hline $25-48$ & 16 & 12.77 & 13.50 & 16.48 \\
\hline TOTAL & 248 & 248 & 248 & 248 \\
\hline $\begin{array}{c}\text { ML } \\
\text { Estimate }\end{array}$ & & $\begin{array}{l}\mathrm{p}=0.854 \\
\mathrm{n}=1.576\end{array}$ & $\begin{array}{c}\mathrm{p}=0.00010775 \\
\beta=5978.5288 \\
\mathrm{n}=29337.08391\end{array}$ & $\begin{aligned} a & =2.0034559 \\
\theta_{1} & =3.8528528 \\
\theta_{2} & =0.0609776\end{aligned}$ \\
\hline $\begin{array}{c}\chi^{2} \\
\text { d.f. }\end{array}$ & & $\begin{array}{c}14.92 \\
17\end{array}$ & $\begin{array}{c}27.79 \\
16\end{array}$ & $\begin{array}{c}11.18 \\
16\end{array}$ \\
\hline
\end{tabular}




\section{QUASI-NEGATIVE-BINOMIAL DISTRIBUTION PROPERTIES}

Table 4.2: Counts of numbers of European red mites on apple leaves; data from Garman, 1951

\begin{tabular}{|c|c|c|c|c|}
\hline \multirow{2}{*}{ Count } & \multirow{2}{*}{$\begin{array}{c}\text { Observed } \\
\text { Frequency }\end{array}$} & NBD & $\begin{array}{c}\text { GNBD Jain \& } \\
\text { Consul's (1971) }\end{array}$ & $\begin{array}{c}\text { QNBD Proposed } \\
\text { Model }\end{array}$ \\
\cline { 3 - 5 } & & 69.49 & 69.49 & 70.91 \\
\hline 0 & 70 & 37.60 & 37.60 & 33.93 \\
1 & 38 & 20.10 & 20.10 & 20.07 \\
3 & 17 & 10.70 & 10.70 & 12.01 \\
4 & 10 & 05.69 & 05.69 & 6.89 \\
5 & 09 & 03.02 & 03.02 & 3.63 \\
6 & 03 & 01.60 & 01.60 & 1.69 \\
7 & 02 & 00.85 & 00.85 & 0.65 \\
8 & 01 & 00.95 & 00.95 & 0.22 \\
\hline TOTAL & 150 & 150 & 150 & 150 \\
\hline \multirow{2}{*}{ ML } & & $\mathrm{p}=0.5281$ & $\mathrm{p}=0.52810$ & $a=0.6268217$ \\
Estimates & & $\mathrm{n}=1.0246$ & $\beta=1.000$ & $\theta_{1}=2.3046227$ \\
\hline$\chi^{2}$ & & 2.484 & $\mathrm{n}=1.0246$ & $\theta_{2}=-0.1785658$ \\
\hline d.f. & & 3 & 2.484 & 2 \\
\hline
\end{tabular}

References

Charalambidies, C. A. (1990). Abel series distributions with applications to fluctuations of sample functions of stochastic processes, Communications in Statistics- Theory and Methods, 19, 317-335.

Consul, P. C. (1974). A simple urn model dependent on predetermined strategy, Sankhya, B, 36, 391-399

Consul, P. C. (1989). Generalized Poisson distributions properties and applications, Marcel Dekker, Inc.: New York, NY.

Consul, P. C., \& Famoye, F. (2006).

Lagrangian Probability Distributions,

Birkhauser: Boston, MA.

Consul, P. C., \& Jain, G.C. (1973). A generalization of the Poisson distribution, Techno-metrics, 15(4), 791-799.

Consul, P. C., \& Shenton, L.R. (1972). Use of Lagrangian expansion for generating discrete generalized probability distributions, SIAM J. Appl. Math., 23(2), 239-248.
Consul, P. C., \& Shenton, L.R. (1974). On the probabilistic structure and properties of discrete Lagrangian distributions, statistical distribution in scientific work, Vol. 1, G.P. Patil, S. Kotz, \& J. K. Ord, Eds. D. Reidel Company: Boston, MA.

Feller, W. (1943): On a general class of contagious distributions, Ann. Math. Stat. 14, 389-400.

Gupta, R. C., \& Ong, S. H. (2004). A new generalization of the negative binomial distribution, Computational Statistics and Data Analysis, 45, 287-300.

Haight, F. A., \& Brever, M. A. (1960). The Borel-Tanner distribution, Biometrika, 47, 143-150.

Holgate, P. (1970). The modality of compound Poisson distribution, Biometrika, 57, 665-667.

Janardan, K. G. (1975). Markov-Polya urn-model with pre-determined strategies, Gujarat Statist. Rev., 2 (1), 17-32. 


\section{HASSAN \& BILAL}

Johnson, N. L., \& Kotz, S. (1992). Univariate Discrete distributions, second edition, John Willy \& Sons, Inc.

Nand, S. B., \& Das, K. K. (1994). A family of Abel series distribution, Sankhya, B, 56(2), 147-164.
Sen, K. \& Jain, R. (1996). Generalized Markov-Polya urn-model with pre-determined strategies. J. Statist. Plann. Infer., 54, 119-133.

Whittaker, E. T., \& Watson, G. N. (1927). A course of modern analysis, Cambridge University Press, Cambridge, MA. 